\title{
Modulation of cross-frequency coupling by novel and repeated stimuli in the primate ventrolateral prefrontal cortex
}

\author{
Joji Tsunada ${ }^{1}{ }^{*}$, Allison E. Baker ${ }^{2}$, Kate L. Christison-Lagay ${ }^{1}$, Selina J. Davis ${ }^{1}$ and Yale E. Cohen ${ }^{1}$ \\ ${ }^{1}$ Department of Otorhinolaryngology - Head and Neck Surgery, University of Pennsylvania School of Medicine, Philadelphia, PA, USA \\ ${ }^{2}$ Department of Neurobiology, Harvard Medical School, Boston, MA, USA
}

\section{Edited by:}

Pascal Belin, University of Glasgow, UK

\section{Reviewed by:}

Kristina Simonyan, Mount Sinai

School of Medicine, USA

Jonas Obleser, Max Planck Institute

for Human Cognitive and Brain

Sciences, Germany

Yukiko Kikuchi, Newcastle University

Medical School, UK

\section{*Correspondence:}

Joji Tsunada, Department of

Otorhinolaryngology: Head and Neck Surgery, University of Pennsylvania School of Medicine, 3400 Spruce - 5 Ravdin, Philadelphia, PA 19104, USA. e-mail: tsunada@mail.med.upenn.edu
Adaptive behavior depends on an animal's ability to ignore uninformative stimuli, such as repeated presentations of the same stimulus, and, instead, detect informative, novel stimuli in its environment. The primate prefrontal cortex (PFC) is known to play a central role in this ability. However, the neural mechanisms underlying the ability to differentiate between repeated and novel stimuli are not clear. We hypothesized that the coupling between different frequency bands of the local field potential (LFP) underlies the PFC's role in differentiating between repeated and novel stimuli. Specifically, we hypothesized that whereas the presentation of a novel-stimulus induces strong cross-frequency coupling, repeated presentations of the same stimulus attenuates this coupling. To test this hypothesis, we recorded LFPs from the ventrolateral PFC (vPFC) of rhesus monkeys while they listened to a novel vocalization and repeated presentations of the same vocalization. We found that the cross-frequency coupling between the gamma-band amplitude and thetaband phase of the LFP was modulated by repeated presentations of a stimulus. During the first (novel) presentation of a stimulus, gamma-band activity was modulated by the theta-band phase. However, with repeated presentations of the same stimulus, this crossfrequency coupling was attenuated. These results suggest that cross-frequency coupling may play a role in the neural computations that underlie the differentiation between novel and repeated stimuli in the VPFC.

Keywords: vocalization, rhesus monkey, neural oscillation, theta band, gamma band

\section{INTRODUCTION}

A key characteristic of adaptive behavior is the ability of animals to ignore repeated "uninformative" stimuli (i.e., repeated presentations of the same stimulus) and, instead, devote neural resources to the detection of novel, and likely more informative, stimuli in the environment. Given the key role of the prefrontal cortex (PFC) in adaptive behavior and executive functions (Miller and Cohen, 2001), it is unsurprising that the PFC plays a central role in this component of behavior (Miller et al., 1996; Ranganath and Rainer, 2003; Grill-Spector et al., 2006; Garrido et al., 2009). Indeed, human patients with prefrontal lesions are noticeably impaired in their ability to detect a novel stimulus that is interspersed among repeated presentations of another stimulus (Daffner et al., 1998, 2000a,b). Importantly, this PFC-lesion phenotype cannot be attributed to simply a sensory or perceptual deficit because lesions in early sensory areas have relatively little effect on novelty detection (Alain et al., 1998). Consistent with this behavioral data, electrophysiological studies indicate that in PFC-lesioned patients, PFC activity does not differentiate between novel and repeated stimuli; whereas in control subjects, novel stimuli generate more PFC activity than repeated stimuli (Knight, 1984; Alho et al., 1994; Daffner et al., 1998, 2000a,b; Hermann and Knight, 2001; Doeller et al., 2003; Haenschel et al., 2005). Together, these findings suggest that the PFC is necessary for novelty detection. However, little is known about how PFC neural activity differentially encodes novel and repeated stimuli.

One possible means by which PFC activity could encode novel versus repeated stimuli is through the interplay between the spiking activity of different neural populations with different tuning profiles (Desimone, 1996; Baldeweg, 2006; Grill-Spector et al., 2006). Specifically, if a neuron is tuned for a particular stimulus, repeated presentations of that stimulus will maintain, or even enhance its firing rate. However, if a neuron is not tuned for that stimulus, repeated presentations of the stimulus will decrease (habituate) this neuron's firing rate. Thus, novel and repeated stimuli can be differentiated by tracking the firing-rate history of neurons that are either tuned or not tuned for a particular stimulus. These differential patterns of spiking activity are thought to depend on their coupling with the phase of the gamma band of the local field potential (LFP; Bragin et al., 1995; Buzsaki et al., 2003; Lakatos et al., 2005; Canolty and Knight, 2010; Moldakarimov et al., 2010a,b).

However, since spiking activity and gamma-band oscillations are often coupled with other frequency bands (Bragin et al., 1995; Lakatos et al., 2005; Lee et al., 2005; Buzsaki, 2006; Osipova et al., 2008; Tort et al., 2008, 2009; Axmacher et al., 2010; Canolty and Knight, 2010; Voytek et al., 2010; Arnal et al., 2011), the neural computations underlying the brain's capacity to distinguish 
between novel and repeated stimuli may also depend on the coupling between multiple frequency bands (e.g., the coupling between the gamma band and the theta or delta bands). Under this hypothesis, novel-stimulus presentation may elicit strong cross-frequency coupling, whereas repeated-stimulus presentations may attenuate this coupling. More specifically, the gammaband response to the novel stimulus may be increased by the bursting phase of other frequency bands, but the gamma-band response to the repeated stimuli may not be modulated by these frequency bands.

Here, we tested whether gamma-band oscillations were coupled to other frequency bands and whether this coupling was modulated by the presentation of novel and repeated stimuli. To test this hypothesis, we recorded LFPs from the ventrolateral PFC (vPFC) of rhesus monkeys while they listened to a vocalization (i.e., the novel stimulus) that was followed by two to four repeated presentations of the same vocalization. We found that the cross-frequency coupling between the gamma-band amplitude and theta-band phase of LFP was attenuated by repeated presentations of a stimulus. These results suggest that the crossfrequency coupling between the gamma and theta oscillations may contribute to a mechanism underlying the brain's capacity to distinguish between novel and repeated stimuli.

\section{MATERIALS AND METHODS SUBJECTS}

Local field potentials and single-unit activity were recorded from two adult male rhesus macaque monkeys (Macaca mulatta; Monkeys $\mathrm{T}$ and $\mathrm{G}$ ). All of the protocols for the surgeries, training, and data collection used in this study were approved by the Dartmouth Institutional Animal Care and Use Committee. The surgical procedures were similar to those used in our previous studies (Cohen et al., 2007, 2009; Russ et al., 2008). Because this study used a passive paradigm, no task-related training was required. However, both monkeys had previous training on auditory and visual tasks.

\section{STIMULI}

The stimuli were auditory- and visual-communication signals from a rhesus monkey. These stimuli were provided by Dr. Asif Ghazanfar and detailed information of these stimuli was provided in previous studies (Lewkowicz and Ghazanfar, 2006; Zangenehpour et al., 2009). Briefly, these signals were obtained from the same monkey; Monkeys $\mathrm{T}$ and $\mathrm{G}$ did not know this monkey. For the auditory signals, we presented one exemplar from three different classes of conspecific vocalizations: a coo, a grunt, and a scream (Figure 1B). Coos and grunts convey the presence of common, low-quality food items (e.g., monkey chow), and are affiliative calls produced in other non-food contexts (e.g., group movement, grooming; Marler et al., 1992; Hauser and Marler, 1993; Hauser, 1998). Screams are elicited when an individual is attacked or threatened (Gouzoules et al., 1984, 1998; Gouzoules and Gouzoules, 2000). The coo had a duration of $735 \mathrm{~ms}$, the grunt had a duration of $180 \mathrm{~ms}$, and the scream had a duration of $215 \mathrm{~ms}$. The sound level of each vocalization was normalized to $65 \mathrm{~dB}$ SPL.

For the visual-communication signals, we presented a silent movie of the monkey producing one of the three vocalizations (the coo, the grunt, or the scream; see Figure 1B). The duration of each movie was $1500 \mathrm{~ms}$. The movie only showed the monkey's head. The dimension of each visual stimulus averaged $12^{\circ}$.

\section{PASSIVE-LISTENING AND -VIEWING PARADIGM}

We developed the passive-listening and -viewing paradigm, which is a modified version of the classic oddball paradigm (Näätänen, 1992), to test how communication signals of one modality modulate communication signals in a different modality. Our analyses focused exclusively on neural response during the passive-listening component of this study; see text at the end of this sub-section for more details.

Specifically, each monkey participated in the passive-listening and -viewing paradigm (Figure 1A) in a darkened, single-walled, sound-attenuated room. During this paradigm, the monkey was seated in a primate chair and had his head restrained to minimize head movements. The chair was placed $1 \mathrm{~m}$ away from a computer monitor (Syncmaster 171T, Samsung); the vocalizations and movies were presented from this monitor. A vocalization was presented three to five times. After the last vocalization was presented, a silent movie was presented. The inter-stimulus interval was 3.1-3.3 s. To keep the monkeys motivated and awake, a juice reward was randomly delivered on $75 \%$ of the trials following movie offset. We also ensured that the monkeys were awake during this paradigm by monitoring the monkeys' eye movements with an infrared camera (Eye-Trac6 RS6-HS, Applied Science Laboratories). Finally, to ensure that long-term adaptation effects were not confounded with short-term adaptation effects (i.e., those effects observed during a trial of the passivelistening and -viewing paradigm), the stimuli were presented to the monkeys for several months prior to the start of the recording sessions.

In each recording session, all the three vocalizations (i.e., the coo, the grunt, and the scream) were presented but only one movie type (e.g., the silent movie of the monkey eliciting the coo) was presented; this movie was randomly selected prior to the start of a recording session. However, on a trial-by-trial basis, the vocalization (e.g., coo), and the number of repeated presentations of this vocalization were randomly varied. Based on this paradigm design, we can consider the movie presentation and the first presentation of the vocalization as "novel" stimuli. The remaining vocalizations can be considered "repeated" stimuli. Our analyses focused exclusively on the responses elicited by the vocalizations since they were presented as both novel and repeated stimuli, unlike the movies, which were not presented as repeated stimuli. We do not present any data on the responses elicited by the movies or any cross-modal effect between the vocalizations and the movies.

\section{RECORDING PROCEDURES}

Neurophysiological recordings were performed with a tungsten microelectrode (1-2 M $\Omega$ at $1 \mathrm{kHz}$; Frederick Haer \& Co.) that was seated in a stainless-steel guide tube. The electrode and guide tube were advanced into the brain with a hydraulic microdrive (MO-95, Narishige). The neural signal was sampled at $24 \mathrm{kHz}$ and band-pass filtered between $2.2 \mathrm{~Hz}$ and $6 \mathrm{kHz}$ with a pre-amplifier (RA16PA, Tucker-Davis Technologies) and an amplifier (RZ2, Tucker-Davis Technologies) and stored for offline analysis. 


\section{A

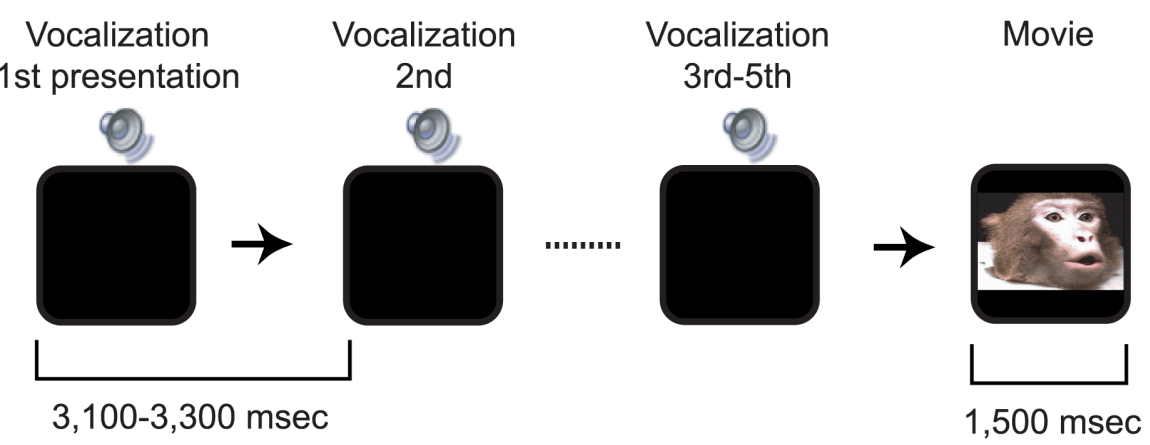

B
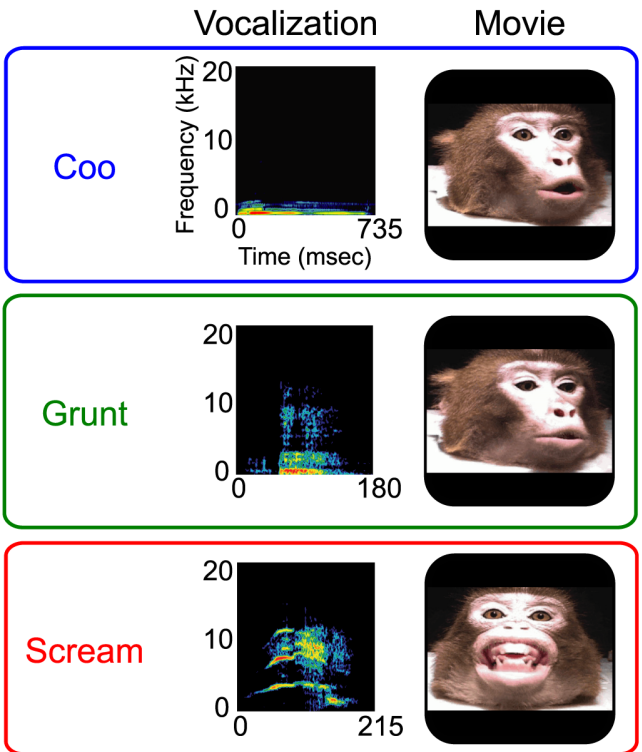

FIGURE 1 | Behavioral paradigm and stimuli. (A) Passive-listening and -viewing paradigm. Each vocalization was presented three to five times with a 3100- to 3300-ms inter-stimulus interval. Following the last presentation of a vocalization, a silent movie of a monkey vocalizing was presented. Within a block of trials, the type of movie remained constant, while the vocalization varied on a trial-by-trial basis. (B) Spectrograms and movie frames of the three classes of vocalizations. The top row shows the spectrogram for the coo and a frame from the movie of the monkey vocalizing the coo. The middle row shows the spectrogram of the grunt and a frame from the movie of the monkey vocalizing the grunt. The bottom row shows the spectrogram of the scream and a frame from the movie of the monkey vocalizing the scream.
Neural activity was recorded from the left vPFC of Monkey T and from the right vPFC of Monkey G. All recording sites were guided by pre- and post-operative magnetic resonance images of each monkey's brain. The vPFC was identified by its anatomical location (i.e., anterior to the arcuate sulcus and Area 8a and below the principal sulcus; areas 45 and 12) and its neurophysiological properties (Romanski and Goldman-Rakic, 2002; Cohen et al., 2004).

Electrodes were advanced into the vPFC until single-unit activity was identified ( $\sim 1-3 \mathrm{~mm}$ from the cortical surface). After single-unit activity was well isolated from a recording site, the monkeys participated in the passive-listening and -viewing paradigm. Neural activity was recorded while the recording properties of the site remained stable; typically, we were able to collect data from $\geq 75$ trials of the paradigm in each recording session.

\section{DATA ANALYSIS}

The LFPs were extracted using methods similar to those described in previous studies (Ghazanfar et al., 2005, 2008; Chandrasekaran and Ghazanfar, 2009). All of the analyses were performed using Chronux $^{1}$ (Mitra and Pesaran, 1999; Bokil et al., 2007) and the EEG toolbox ${ }^{2}$ (Rizzuto et al., 2003, 2006) that ran on the Matlab (The MathWorks Inc.) programming platform.

Neural activity was first low-pass filtered with a $300-\mathrm{Hz}$ cutoff frequency using a four-pole bidirectional Butterworth filter. Next, the filtered signals were resampled at $1 \mathrm{kHz}$. When necessary, $60-\mathrm{Hz}$ (line) noise was removed from the signals. We

\footnotetext{
${ }^{1}$ http://www.chronux.org

${ }^{2}$ http://memory.psych.upenn.edu/Software
} 
analyzed three different types of LFP signals: the "total"-LFP power, stimulus-evoked LFP, and induced LFP. Total-LFP power, which reflects both phase-locked and non-phase-locked neural signals, was obtained by calculating the power spectrum from individual trials with a Morlet-wavelet decomposition (Sinkkonen et al., 1995; Tallon-Baudry et al., 1996; Mallat, 1999; Samar, 1999; Tallon-Baudry and Bertrand, 1999; Ghazanfar et al., 2008; Chandrasekaran and Ghazanfar, 2009; Canolty and Knight, 2010) and then averaging these spectra (Tallon-Baudry and Bertrand, 1999; Lakatos et al., 2007; Ghazanfar et al., 2008; Chandrasekaran and Ghazanfar, 2009). The stimulus-evoked LFP, which reflects phase-locked neural signals, was obtained by averaging the LFPs across trials, relative to stimulus onset (Makeig et al., 2004; Siegel et al., 2009). The induced LFP, which reflects the non-phase-locked neural signals, was obtained by subtracting the stimulus-evoked LFP from each trial's LFP. Finally, the average of these subtracted signals was calculated (Makeig et al., 2004; Siegel et al., 2009).

The LFPs were divided into different analysis periods. The "baseline" period was a 250-ms period that preceded the onset of the first vocalization of each trial. For the grunt and scream, since reliable increases in power were evident from vocalization onset through vocalization offset, the "stimulus" period for these vocalizations was their entire duration. However, since reliable increases in power were limited to the initial 300-ms for the coo (see Figure 3), the stimulus period for the coo was this $300-\mathrm{ms}$ period. On a site-by-site basis, a $t$-test tested whether the mean total-LFP power of a vPFC site was reliably different during the vocalization period than during the baseline period. Sites for which the null hypothesis $(p<0.05)$ was rejected were defined as "auditory-modulated."

The amplitudes of the band-limited signals of the induced and total LFPs were tested by first applying Butterworth filters with different pass-bands $(4-10 \mathrm{~Hz}$ filter for the theta band; $10-15 \mathrm{~Hz}$ filter for the alpha band; $15-25 \mathrm{~Hz}$ filter for the beta band; and 25$50 \mathrm{~Hz}$ filter for the gamma band) and then by applying a Hilbert transformation to calculate the envelope of these band-limited signals (Chandrasekaran and Ghazanfar, 2009).

The phase-locking of the total-LFP across trials was calculated using two different approaches (Tallon-Baudry et al., 1996; Lakatos et al., 2007, 2009; Ghazanfar et al., 2008). In the first approach, we treated the wavelet-transformed data $w(t, f)^{*} s_{i}(t)$ of each trial $i$ at each frequency $f$ - time $t$ point as a unit vector $P_{i}(t, f): P_{i}(t, f)=w(t, f)^{*} s_{i}(t) /\left|w(t, f)^{*} s_{i}(t)\right|$. Here, $w(t, f)^{*} s_{i}(t)$ indicates the results of the convolution of a Morlet wavelet $w(t, f)$ with the signal $s_{i}(t)$. Next, the average of these $N$ trial-by-trial unit vectors was calculated: $P(t, f)=(1 / N) \Sigma w(t, f)^{*} s(t) /\left|w(t, f)^{*} s(t)\right|$. The degree of phase-locking (i.e., the phase-locking "value") was quantified by calculating the resultant length of this vector; phaselocking values range between 0 and 1 . Higher values indicate that the individual phase values are more clustered than lower values.

In the second approach, we were interested only in the phaselocking of the band-limited signals (e.g., the theta band). To test the phase-locking of these signals, for each trial, we first applied Butterworth filters to the LFPs with different pass-bands and then applied a Hilbert transformation to these filtered signals to obtain a unit vector at each time point. We then averaged these trial-by-trial unit vectors. The phase-locking value was calculated using a procedure analogous to the one described above.

To quantify the effect that the repeated presentations of a vocalization had on the LFPs, we calculated a modulation index. The general form of the index was: $\left(\mathrm{P}^{1 s t}-\mathrm{P}^{2 n d}\right) /\left(\mathrm{P}^{1 s t}+\mathrm{P}^{2 n d}\right)$. $\mathrm{P}$ was either the peak amplitude or the peak phase-locking value of the band-limited signals. The superscript "1st" or "2nd" refers to whether these values were obtained from the first or second presentation of a vocalization, respectively. The value of the index ranges between -1 and 1 . If the index value is $>0$, it indicates that the $\mathrm{P}^{1 \text { st }}$ value was greater than the $\mathrm{P}^{2 \text { nd }}$. If the index value is $<0$, it indicates that the $\mathrm{P}^{2 \mathrm{nd}}$ value was greater than the $\mathrm{P}^{1 \mathrm{st}}$.

Next, the hypothesis that a novel-stimulus induces crossfrequency coupling between the gamma-band amplitude and the phase of other frequency bands of the LFPs was tested (Bragin et al., 1995; Buzsaki et al., 2003; Lakatos et al., 2005; Lee et al., 2005; Osipova et al., 2008; Tort et al., 2008, 2009, 2010; Axmacher et al., 2010; Canolty and Knight, 2010; Voytek et al., 2010). In the first analysis, we tested the cross-frequency coupling between frequency bands of the total LFP. On a trial-by-trial basis, we calculated the peak amplitude of the gamma band and identified the phase of the theta, alpha, and beta bands at which the gamma-band peak occurred. Then, on a site-by-site basis, these phase values were binned to form a distribution of phase (i.e., percentage of trials per phase bin); similar to previous studies, six phase bins were chosen to test the phase distribution (Lakatos et al., 2005, 2007, 2009; Kayser et al., 2008). A permutation test examined whether this measured distribution was reliably different from a shuffled distribution. First, on a site-by-site basis, we shuffled the relationship between the percentages of trials and their phase bins and then averaged the percentage of trials across sites for each phase bin. Next, we generated the resultant vector from these averaged trials and calculated its length. We repeated this procedure 1000 times to generate a shuffled distribution of vector length. If the length of measured vector was $>95 \%$ of the shuffled distribution, we rejected the hypothesis that the measured and shuffled distributions were the same at $p<0.05$.

In a second analysis, we tested the cross-frequency coupling between the frequency bands of the induced LFP. On a trial-bytrial basis, we calculated the amplitude of the gamma band during the first $300 \mathrm{~ms}$ following stimulus onset. Next, on a site-by-site basis, we formed distributions of the gamma-band amplitude as a function of the phase of the theta, alpha, or beta bands; similar to the previous analysis, the amplitude distributions were divided amongst six phase bins. A permutation test examined whether our measured amplitude distribution was reliably different from a shuffled distribution. We used a permutation test that is analogous to the one described above except that we used amplitude instead of percentage of trials.

\section{RESULTS}

We recorded the LFPs from 168 recording sites in the vPFC of two rhesus monkeys (monkey T: 103 sites, monkey G: 65 sites). Neural data were recorded while the monkeys listened to three to five repeated presentations of a vocalization. Of the 168 sites, we found that total-LFP power increased at 79 sites (monkey T: 59 
sites, monkey G: 20 sites) during the presentation of at least one type of vocalization (i.e., auditory-modulated sites).

\section{THE AMPLITUDE OF THE STIMULUS-EVOKED LFP DECREASED WITH REPEATED PRESENTATIONS OF VOCALIZATIONS}

Figure 2 illustrates the stimulus-evoked LFP in response to repeated presentations of vocalizations; since there were relatively fewer trials that had four or five presentations of a vocalization and since the neural responses to the fourth and fifth presentations were comparable, these data were grouped together. These stimulus-evoked LFPs mainly reflect the neural activity that is phase-locked to stimuli or task-related events. The top row shows the stimulus-evoked LFP recorded from a single recording site as

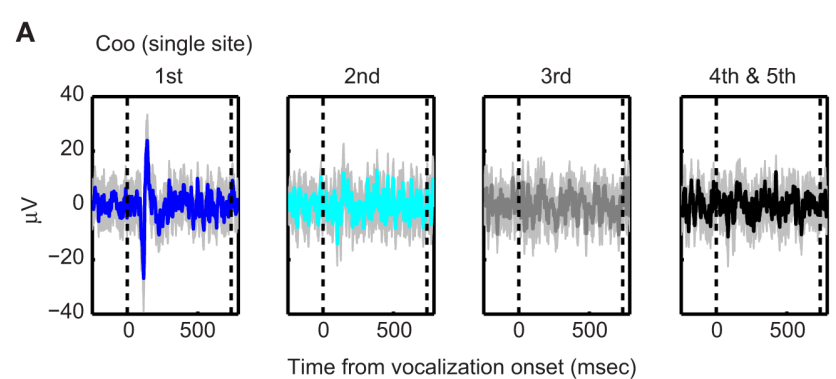

B
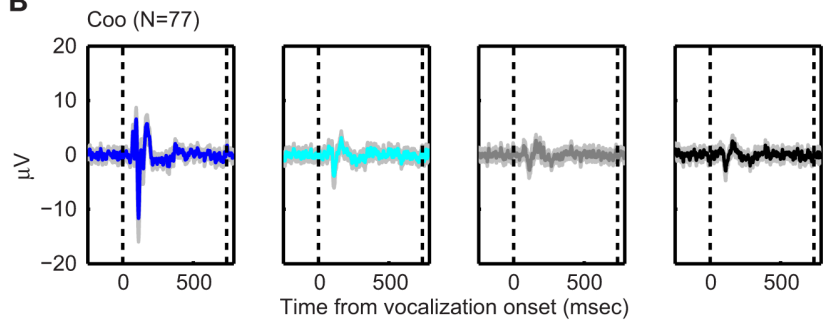

C

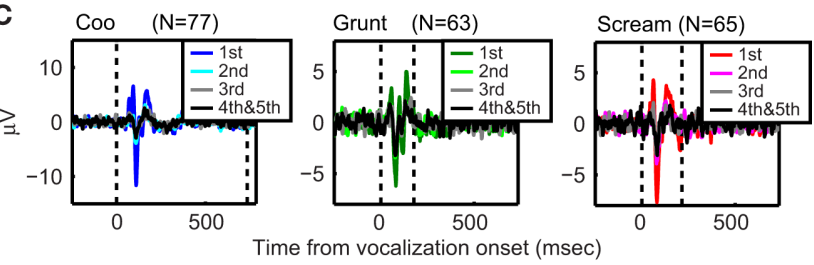

FIGURE 2 |The stimulus-evoked LFP decreased with repeated presentations of a stimulus. The data in (A) show an example from a single site (1st-3rd: $n=25$ trials, 4th and 5th: $n=22$ trials); whereas those in $\mathbf{( B , C )}$ show population-level stimulus-evoked LFP. In (A, $\mathbf{B})$, the data illustrate the effect that repeated presentations of the coo vocalization had on the LFP. The data in the first column illustrate the LFPs from the first vocalization presentation. The data in the rightmost column illustrates the LFPs from the fourth and fifth vocalization presentations; the data from fourth and fifth presentations were combined. The gray error bars are bootstrapped $95 \%$ confidence intervals of mean. The data in (C) illustrate how the stimulus-evoked LFP decreased as a function of stimulus presentation. The data in each column were generated with a different vocalization: coo (left; $n=77$ sites), grunt (middle; $n=63$ sites), and scream (right; $n=65$ sites). Within each plot, the stimulus-evoked LFP are plotted as a function of stimulus presentation. All of the data are aligned relative to vocalization onset (i.e., the vertical dotted line at time $t=0$ ). The second dotted line indicates vocalization offset a function of repeated presentations of the coo vocalization. The middle row shows the mean stimulus-evoked LFP calculated from the population of coo responsive sites. The bottom row shows the mean stimulus-evoked LFP as a function of the coo, grunt, and scream and as a function of stimulus repetition of each of these vocalizations. These data suggest that the amplitude of the stimulus-evoked LFP decreased with repeated presentations for all three vocalizations. Finally, the modulation of the stimulus-evoked LFP was observed throughout the entire period of data collection (i.e., across many months), which indicates that there was not any long-term adaptation to the stimuli.

\section{THE TOTAL-LFP POWER AND PHASE-LOCKING VALUES DECREASED WITH REPEATED PRESENTATIONS OF VOCALIZATIONS}

Figures 3A,B illustrates the relationship between total-LFP power, vocalization, and the number of vocalization presentations. As can be seen, independent of the number of stimulus presentations, the coo modulated the total-LFP power across a broad range of frequencies $(4-50 \mathrm{~Hz}$; Figure $3 \mathbf{A})$. These frequencies spanned the theta, alpha, beta, and gamma bands. LFP power across these frequencies, on average, decreased with sequential presentations of the coo vocalization (Figure 3A). Similar reductions in LFP power were identified for repeated presentations of the grunt and the scream vocalizations (Figure 3B). The time-amplitude profile of the gamma band is shown in Figure 4A. As expected from the data shown in Figure 3, the gamma-band amplitude decreased with stimulus presentation. These time-amplitude profiles also indicate that the greatest decrease in amplitude was between the first and second stimulus presentations.

To quantify these observations, we calculated the mean and peak amplitude of different band-limited LFPs as a function of the number of repeated vocalizations. We found that both the mean and the peak amplitude of the gamma-, as well as the theta-, alpha-, and beta-bands, decreased reliably between the first and second vocalization presentations (one-way ANOVA with post hoc Tukey-Kramer tests, $p<0.05)$. However, the time-amplitude profile during the third vocalization presentation was not reliably different than that seen during the fourth/fifth vocalization presentations. To assess the magnitude of the amplitude reduction between the first and second stimulus presentations, we computed a modulation index; see Section "Materials and Methods" for more details. Figure 4B shows the distributions of modulation-index values for the gamma band as a function of vocalization. The mean value of each distribution (coo: 0.05 ; grunt: 0.03 ; scream: 0.03 ) was reliably greater than 0 ( $t$-test, $p<0.05$ ), indicating that, on average, the amplitude of the gamma band decreased between the first and second vocalization presentation; analogous results were found for the other frequency bands (data not shown).

Next, we tested the hypothesis that the phase-locking of the LFPs attenuated with repeated presentations of a vocalization (Jansen et al., 2003; Fuentemilla et al., 2006). To test this hypothesis, we calculated the extent to which the LFPs were phase-locked across trials by normalizing the stimulus-period phase-locking values relative to the baseline phase-locking values (Tallon-Baudry et al., 1996; Lakatos et al., 2007, 2008; Ghazanfar et al., 2008). After the onset of the first stimulus, the phase-locking values between 4 and $50 \mathrm{~Hz}$ were greater than those observed during the baseline 
A
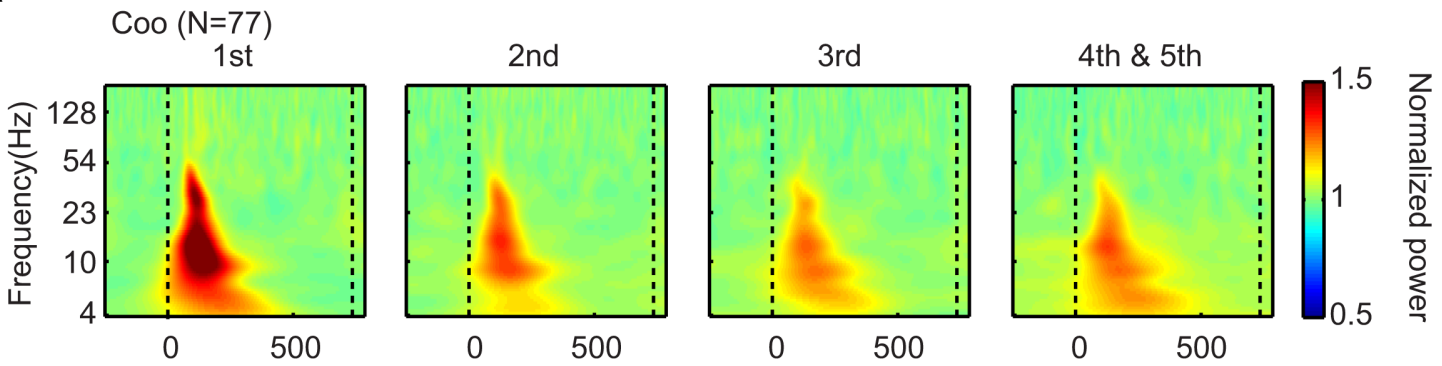

B

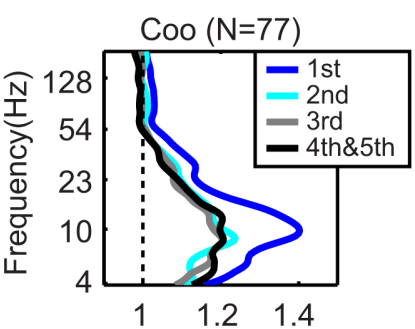

C

Time from vocalization onset ( $\mathrm{msec})$
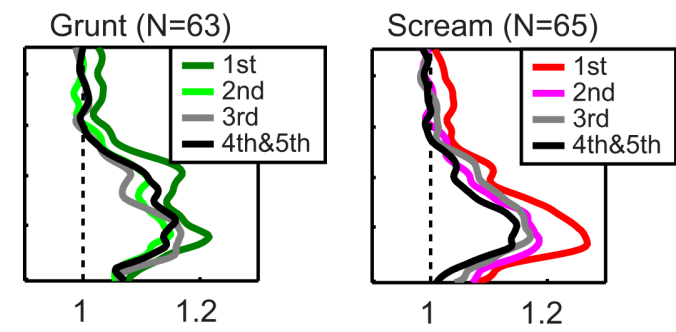

Normalized power
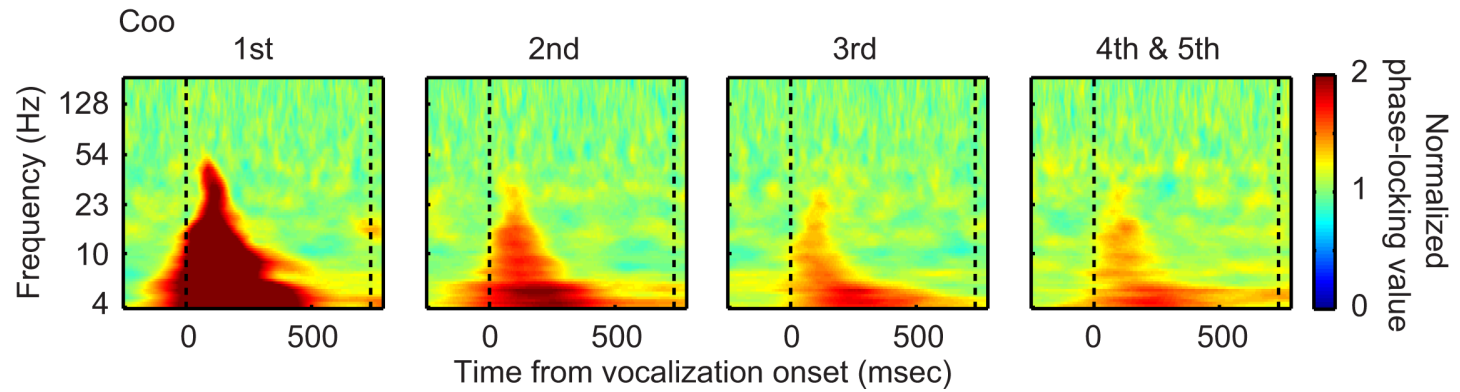

D
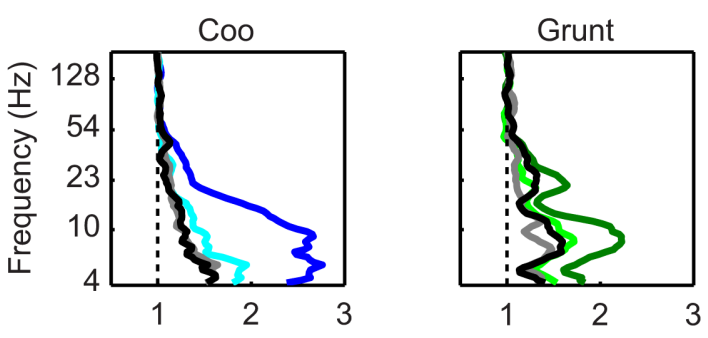

Normalized phase-locking value

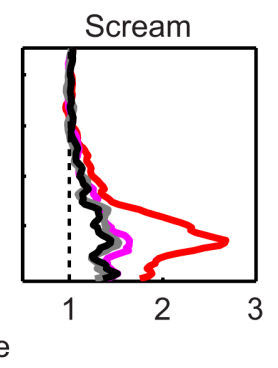

FIGURE 3 | Total-LFP power and phase-locking decreased with repeated presentations of a vocalization. The data in $(\mathbf{A})$ illustrate the effect that repeated presentations of the coo vocalization had on the population spectrogram. The data in the first column illustrate the population spectrogram of total-LFP power from the first vocalization presentation. The data in the rightmost column illustrates the population spectrogram from the fourth and fifth vocalization presentations; the data from fourth and fifth presentations were combined. All of the data are aligned relative to vocalization onset (i.e., the vertical dotted line at time $t=0)$. The second dotted line indicates vocalization offset. The data in (B) illustrate the effect that repeated presentations of a vocalization had on the population spectra. The data in each column were generated from a different vocalization: coo (left; $n=77$ sites), grunt (middle; $n=63$ sites), and scream (right; $n=65$ sites). Within each plot, the spectra are plotted as a function of stimulus presentation. The data in (C,D) illustrate the effect that repeated presentations of a vocalization had on phase-locking; the data in these two panels are organized analogously to that described in $(\mathbf{A}, \mathbf{B})$, respectively. period (Figures 3C,D). These phase-locking values decreased with repeated-stimulus presentations but remained reliably greater than baseline values (Figures 3C,D). In contrast, at higher frequencies (i.e., $>50 \mathrm{~Hz}$ ), the phase-locking values were not reliably different than those observed during the baseline period. The temporal profile of phase-locking in the theta band is shown in Figure 5A. Consistent with the data shown in Figures 3C,D, phaselocking decreased with repeated presentations of a vocalization with the greatest decrease in phase-locking seen between the first and second stimulus presentations (Figure 5A). 

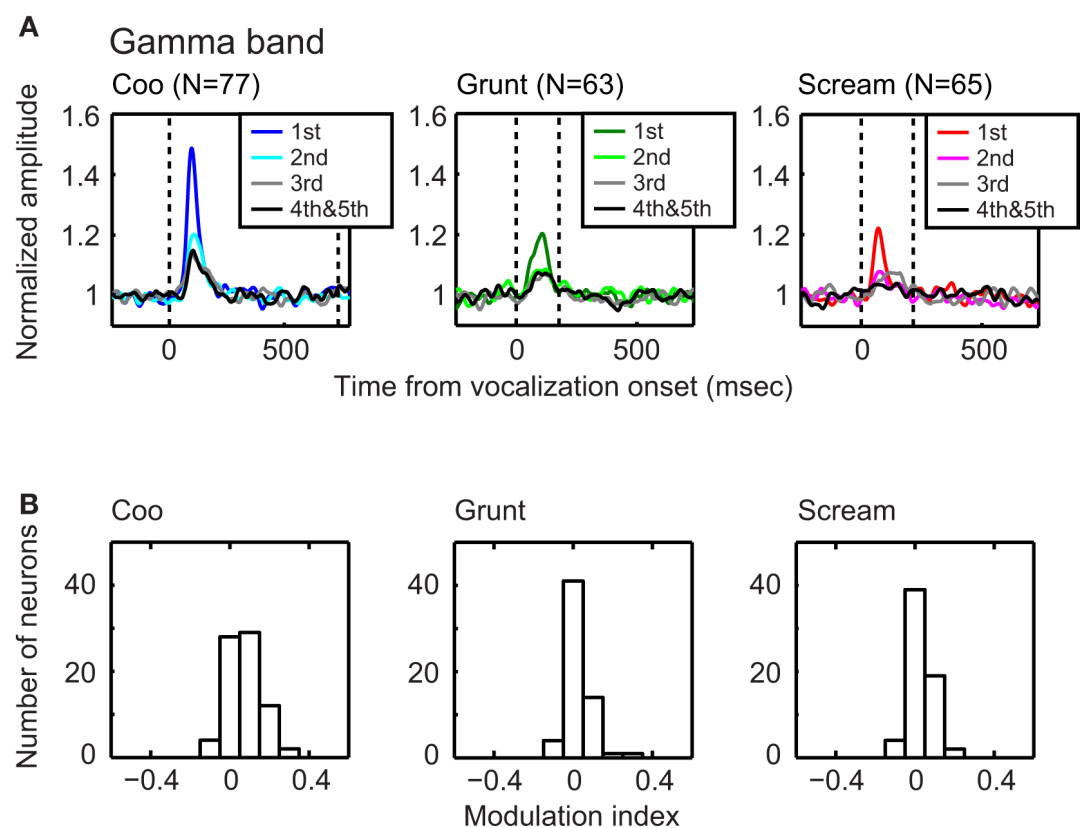

FIGURE 4 |The gamma-band $(25-50 \mathrm{~Hz})$ amplitude of the total LFP decreased with repeated presentations of a vocalization. The data in each column of $(\mathbf{A})$ illustrate the effect that repeated presentations of a vocalization had on the normalized amplitude of the gamma band. The data in each column were generated from a different vocalization: coo (left; $n=77$ sites), grunt (middle; $n=63$ sites), and scream (right; $n=65$ sites). Within each plot, the amplitude-time profiles are plotted as a function of stimulus presentation. Data are aligned relative to vocalization onset (i.e., the vertical dotted line at time $t=0$ ). The second dotted line indicates vocalization offset. The data in (B) show the distributions of modulation-index values as a function of vocalization: coo (left; $n=77$ sites), grunt (middle; $n=63$ sites), and scream (right; $n=65$ sites). The mean value of each distribution is reliably ( $t$-test; $p<0.05)$ greater than zero, indicating that, for each vocalization, the amplitude of the gamma band reliably decreased with repeated presentations of the stimulus.
To quantify these observations, we calculated the peak phaselocking value as a function of the number of repeated vocalizations. We found that the peak phase-locking value of the gamma-, as well as the theta-, alpha-, and beta-bands, decreased reliably between the first and second vocalization presentations (one-way ANOVA with post hoc Tukey-Kramer tests, $p<0.05)$. However, the time-amplitude profile from the third vocalization presentation was not reliably different than that seen on the fourth/fifth vocalization presentations. To assess the magnitude of the phase-locking reduction between the first and second stimulus presentation, we computed a modulation index; see Section "Materials and Methods" for more details. Figure 5B shows the distributions of modulation-index values for the theta band as a function of vocalization. The mean value of each distribution (coo: 0.20 ; grunt: 0.07 ; scream: 0.19 ) was reliably greater than 0 ( $t$-test, $p<0.05$ ), indicating that, on average, the phase-locking of the theta-band decreased between the first and second vocalization presentation; analogous results were found for the other frequency bands (data not shown).

\section{THE CROSS-FREQUENCY COUPLING BETWEEN THE GAMMA-BAND AMPLITUDE AND THE THETA-BAND PHASE WAS ATTENUATED WITH REPEATED PRESENTATIONS OF VOCALIZATIONS}

Next, we tested the hypothesis that the peak amplitude of the gamma band occurred at specific phases of other LFP frequency bands. Moreover, we hypothesized that this relationship between the gamma and other frequency bands was strongest during the first-stimulus (i.e., the novel stimulus) presentation and decreased with repeated presentations of a vocalization.

To test these hypotheses, we analyzed the cross-frequency coupling between frequency bands of the total LFP. Figure 6A shows the relationship between the peak amplitude of gamma band and the phase of the theta band at the population level. During the firststimulus presentation, the distribution of theta-band phase was not uniform: the peak amplitude of the gamma band occurred at specific phases of the theta band (Figure 6A, permutation test; coo: $p<0.05$; grunt: $p<0.05$; scream: $p<0.05)$. However, with further stimulus presentations, the peak amplitude of the gamma band did not occur at specific theta-band phases. Instead, it was uniformly distributed across all phase values (permutation test, $p>0.05$ ). Reliable coupling was also identified between the peak amplitude of the gamma band and other frequency bands during the firststimulus presentation. However, this coupling was only present in a subset of the vocalizations (i.e., in the alpha band for the coo, Figure 6C; and in the beta band for the grunt, Figure 6D) and not all three vocalizations like that observed between the gamma and theta bands.

The cross-frequency coupling between the peak amplitude of the gamma band and theta-band phase may not simply be a function of stimulus presentation. Indeed, we speculated that the theta-band phase may also covary with the magnitude of the gamma band's peak amplitude (Lakatos et al., 2005, 2008). To test 
A Theta band

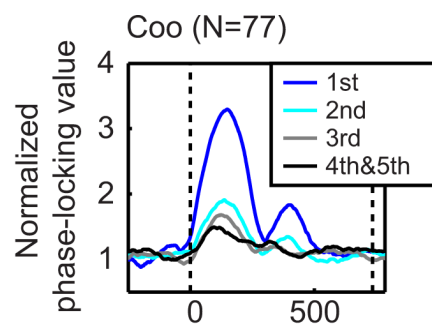

Grunt (N=63)

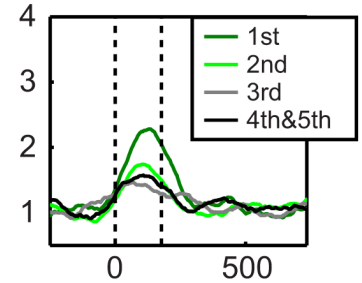

Scream $(\mathrm{N}=65)$

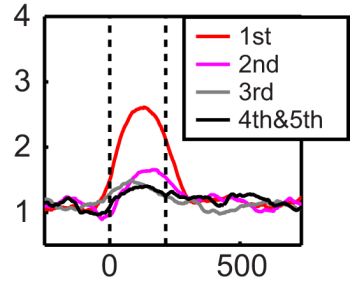

Time from vocalization onset (msec)
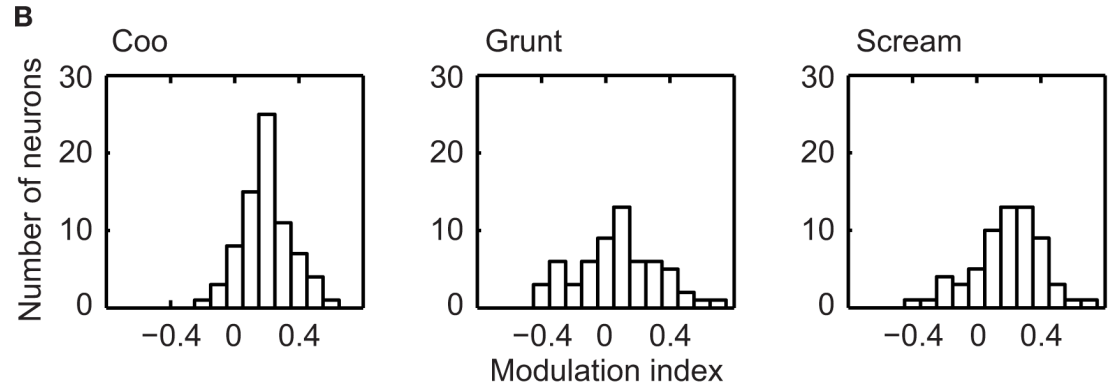

FIGURE 5 |The phase-locking value of the theta band $(4-10 \mathrm{~Hz})$ decreased with repeated presentations of a vocalization. The data in each column of (A) illustrate the effect that repeated presentations of a vocalization had on phase-locking. The data in each column were generated from a different vocalization: coo (left; $n=77$ sites), grunt (middle; $n=63$ sites), and scream (right; $n=65$ sites). Within a plot, the phase-locking values versus time profiles are plotted as a function of stimulus presentation. Data are aligned relative to vocalization onset (i.e., the vertical dotted line at time $t=0$ ) The second dotted line indicates vocalization offset. The data in (B) show the distributions of modulation-index values as a function of vocalization: coo (left; $n=77$ sites), grunt (middle; $n=63$ sites), and scream (right; $n=65$ sites). The mean value of each distribution is reliably ( $t$-test; $p<0.05$ ) greater than zero, indicating that, for each vocalization, the phase-locking of the theta band reliably decreased with stimulus presentation. this idea, on a site-by-site basis, we compared the peak amplitude of the gamma band that occurred at the most common theta-band phase with the peak amplitude of the gamma band that occurred at the least common phase; this analysis was limited to the data generated during the first-stimulus presentation (Figure 6B). We found that the gamma-band amplitude was reliably greater at the most common phase than at the least common phase ( $t$-test, $p<0.05$ ).

However, since the aforementioned analyses used the total LFP (which includes components of both the stimulus-evoked and induced LFP), our findings may simply reflect the inherent phaselocking that occurs with stimulus-evoked activity and the neural habituation that normally occurs with repeated-stimulus presentations. To further explore this issue, we tested the cross-frequency coupling between bands of the induced LFP. Similar to the results from the total LFP, we found that the gamma-band amplitude of the induced-LFP decreased with stimulus repetition (Figure 7A). The greatest decrease in amplitude occurred between the first and second stimulus presentations. In contrast, the band-limited amplitudes of the other frequency bands (i.e., theta, alpha, beta bands) were not modulated by repeated-stimulus presentations; Figure 7B shows the results for the theta band.

Next, we examined the cross-frequency coupling between bands of the induced LFP. Figure $\mathbf{8 A}$ shows the relationship between the gamma-band amplitude and the phase of the theta band at the population level. During the first-stimulus presentation, the amplitude of the gamma band was modulated by the theta phase. That is, the largest amplitude of the gamma band occurred at specific phases of the theta band (Figure $\mathbf{8 A}$, permutation test; coo: $p<0.05$; grunt: $p<0.05$; scream: $p<0.05$ ); the phases for largest gamma-band amplitudes were distributed between $-5 / 6^{*} \pi$ and $-\pi / 2$. Also, unlike the coupling seen with the total LFP (see Figure 6A), reliable coupling during the second stimulus presentation was also seen for the grunt and the scream, but not for the coo (Figure 8A, permutation test; coo: $p>0.05$; grunt: $p<0.05$; scream: $p<0.05)$. However, after the second presentation, the amplitude of the gamma band was not reliably modulated by the theta phase (permutation test, $p>0.05$ ). Reliable coupling between the gamma and alpha bands was seen for the grunt (Figure 8B) but not for the coo and for the scream; we could not identify any reliable coupling between the gamma and beta bands (Figure 8C). Thus, for both the total and induced LFPs, only the coupling between gamma band and theta band was significant for all three vocalizations during the first-stimulus presentation.

\section{DISCUSSION}

We found that the magnitude and the phase-locking of the LFPs in the $\mathrm{vPFC}$ decreased with repeated presentations of a vocalization. Additionally, the cross-frequency coupling between the gamma and theta bands was attenuated by repeated presentations of a vocalization. Specifically, during the first (novel) presentation of a stimulus, the peak of the gamma-band activity occurred 


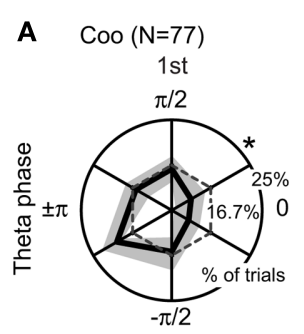

2nd

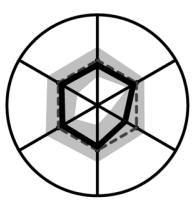

Grunt (N=63)

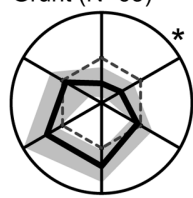

Scream $(\mathrm{N}=65)$
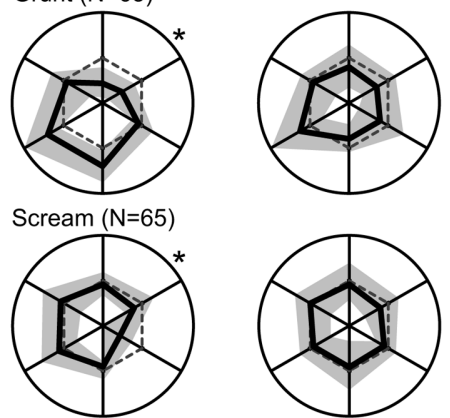
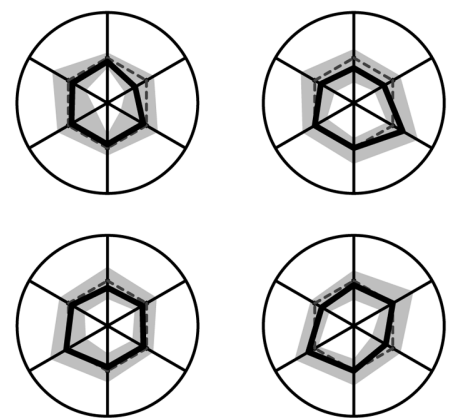

- Measured value

$95 \%$ confidence interval

---- Uniform distribution (16.7\%)

B

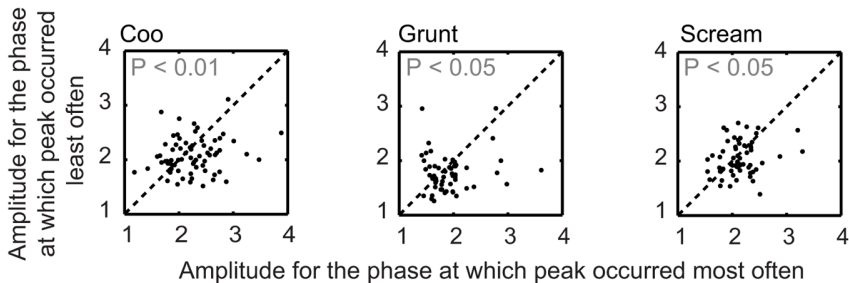

C

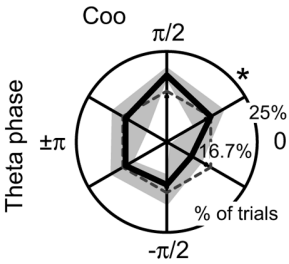

Grunt

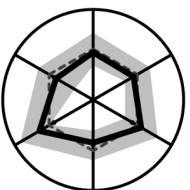

Scream

D

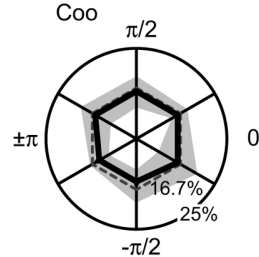

Grunt
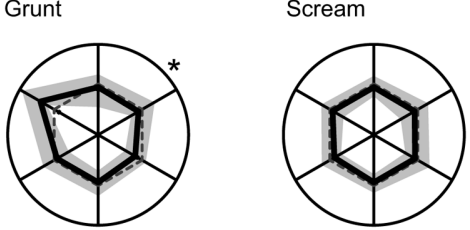

FIGURE 6 |The cross-frequency coupling between the theta phase and gamma-band peak amplitude of the total-LFP attenuated with repeated presentations of a vocalization. The data in $(\mathbf{A})$ show the effect that repeated presentations of a vocalization had on the distribution of the theta phase at the gamma-band peak time. The data in each row show the distributions of phase as a function of vocalization, whereas the data in the columns illustrate the phase distribution as a function of stimulus presentation. In each plot, the solid black line shows the measured phase distribution; the gray area surrounding the black line indicates the $95 \%$ confidence interval of the measured phase distribution; and the dotted line illustrates the uniform distribution of phase (i.e., 16.7\%). Asterisks indicate when phase distribution was not uniform at $p<0.05$. The scatter plots in (B) illustrate, on a site-by-site

basis and as a function of vocalization, the relationship between the mean peak amplitude of the gamma band at the most and least common theta phase; these values were extracted only from data elicited by the first vocalization presentation. The dotted line is the line of equal peak amplitude (i.e., a line with a slope of 1). On average, the peak amplitude of the gamma band at the most common phase was reliably $(p<0.05)$ larger than that at the least common phase. The plots in (C) show the distribution of the alpha phase at the gamma-band peak time during the first presentation of each vocalization. The plots in (D) show the distribution of the beta phase at the gamma-band peak time during the first presentation of each vocalization. For the plots in $(\mathbf{C}, \mathbf{D})$, the solid black line, the gray area surrounding the black line, dotted lines, and the asterisks are in the same format as those described in (A). 


\section{A Gamma band}
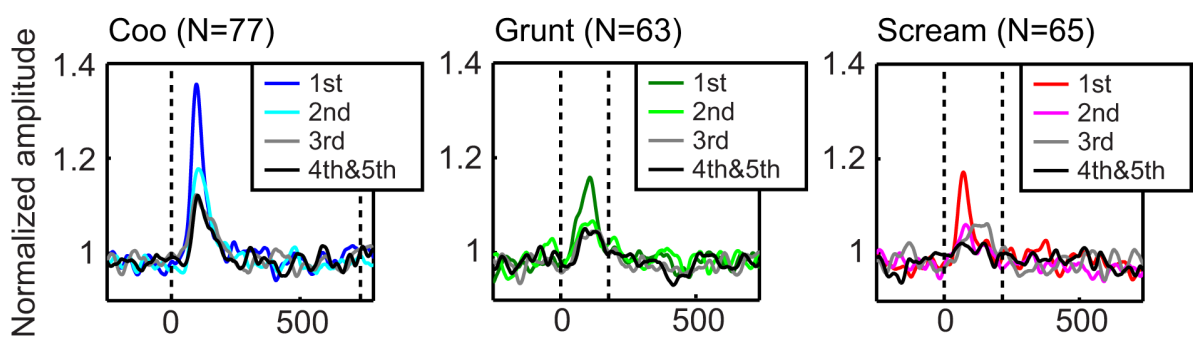

Time from vocalization onset (msec)

B Theta band
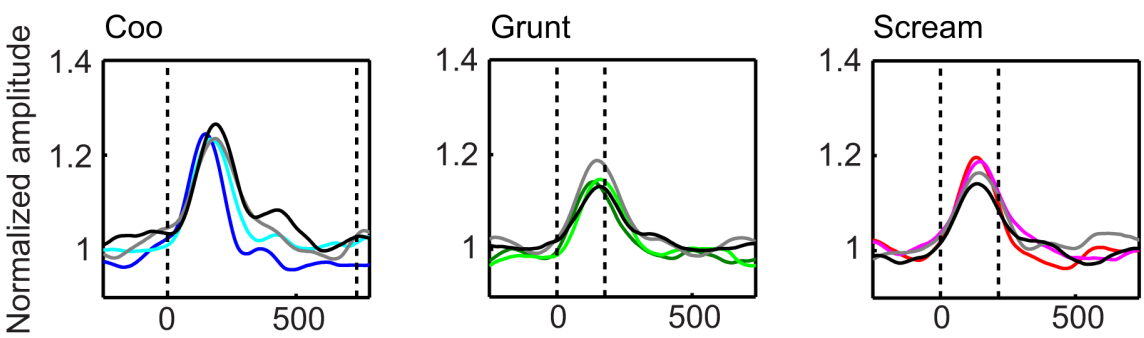

Time from vocalization onset (msec)

FIGURE 7 |The gamma-band, but not the theta-band, amplitude of the induced LFP decreased with repeated presentations of a vocalization. The data in each column of (A) illustrate the effect that repeated presentations of a vocalization had on the normalized amplitude of the gamma band. The data in each column were generated from a different vocalization: coo (left; $n=77$ sites), grunt (middle; $n=63$ sites), and scream (right; $n=65$ sites). The presentation format is same as Figure 4A. The data in each column of (B) illustrate the effect that repeated presentations of a vocalization had on the normalized amplitude of the theta band. most often at specific theta-band phases, and these specific phases induced large-amplitude gamma oscillations, relative to other phases; see Figures 6 and 8. However, with repeated-stimulus presentations, the cross-frequency coupling between the gammaband amplitude and the theta-band phase was attenuated. Thus, our findings suggest cross-frequency coupling may play a role in the neural computations that underlie the differentiation between novel and repeated stimuli in the vPFC.

\section{COMPARISON BETWEEN VOCALIZATION-RESPONSIVE LFPS IN THE vPFC AND THOSE IN EARLIER AUDITORY AREAS}

In the vPFC, the theta, alpha, beta, and gamma bands of the LFPs were modulated by all three vocalizations (Figure 3 ). Since we used only three vocalizations, it is possible that other vocalizations may elicit LFP responses with different spectral profiles. However, because the three acoustically distinct vocalizations (see Figure 1B) elicited similar spectral responses, it is reasonable to assume that other vocalizations would also elicit similar spectral responses. Also, since we only used vocalizations, we cannot conclude that the LFP response is specific to vocalizations. However, by comparing our results with previous vocalization studies, we can discuss how LFP activity in the vPFC is similar to or different from that seen in other brain regions.

Similar to our results in the vPFC, previous studies have found that vocalizations modulate low-frequency $(<20 \mathrm{~Hz})$ LFPs in both the auditory cortex and the superior temporal sulcus (Ghazanfar et al., 2008; Kayser et al., 2008; Chandrasekaran and Ghazanfar, 2009). However, unlike the vPFC, high gamma-band $(>50 \mathrm{~Hz})$ activity is reported in the auditory cortex and the superior temporal sulcus; relatively more high gamma-band activity is seen in the auditory cortex than in the superior temporal sulcus. These results suggest that high gamma-band activity may be area specific and most evident in the early sensory cortex (Chandrasekaran and Ghazanfar, 2009).

\section{COMPARISONS WITH PREVIOUS EEG/LFPs STUDIES THAT USED REPEATED-PRESENTATION PARADIGMS}

In general, our finding that repeated presentations of an auditory stimulus reduces the power and phase-locking of the LFP is consistent with a large body of literature that has examined this issue in early regions of the auditory cortex (Rosburg, 2004; Baldeweg, 2006; Fuentemilla et al., 2006). However, whereas we found reductions in power and phase-locking across a broad band of frequencies (i.e., the theta-, alpha-, beta-, and gamma-frequency bands), similar reductions in early auditory areas are limited to a narrower range of frequencies. For example, EEG studies of the auditory cortex have linked reductions in power and phase-locking with the theta and alpha bands (Jansen et al., 2003; Fuentemilla et al., 2006). Our finding that reductions in power and phase span multiple frequency bands may reflect the involvement of the vPFC in multiple circuits and neural processes (Buzsaki, 2006; Chandrasekaran and Ghazanfar, 2009) in addition to its role in 

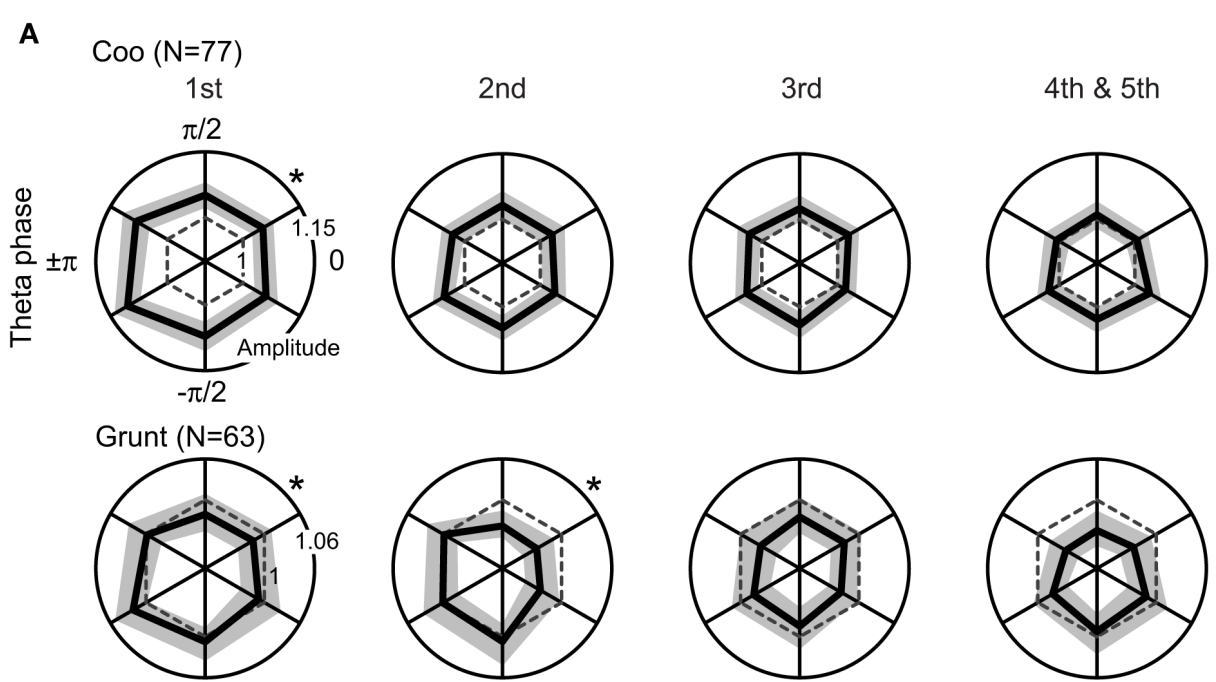

Scream $(\mathrm{N}=65)$
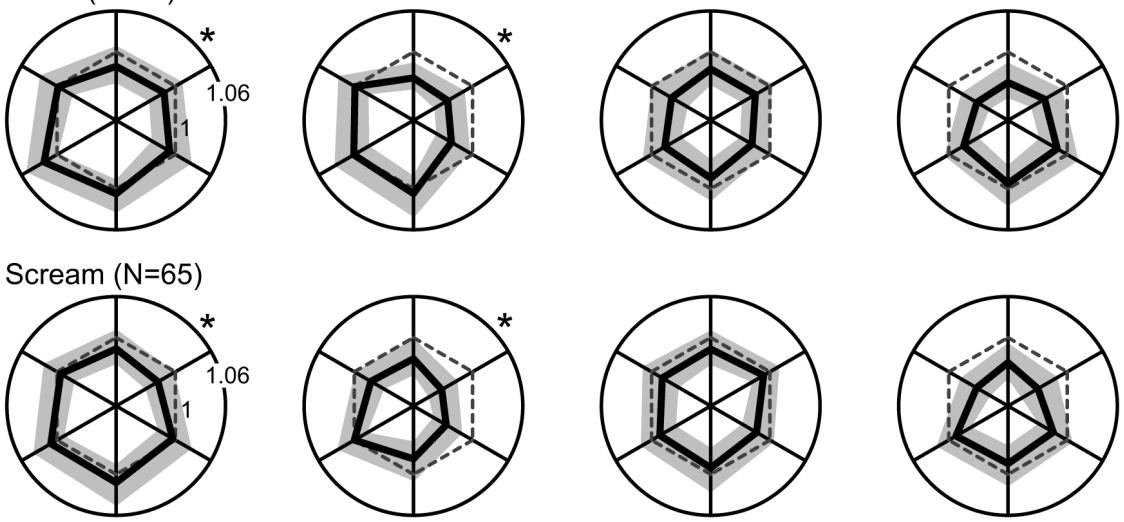

Measured value

$95 \%$ confidence interval
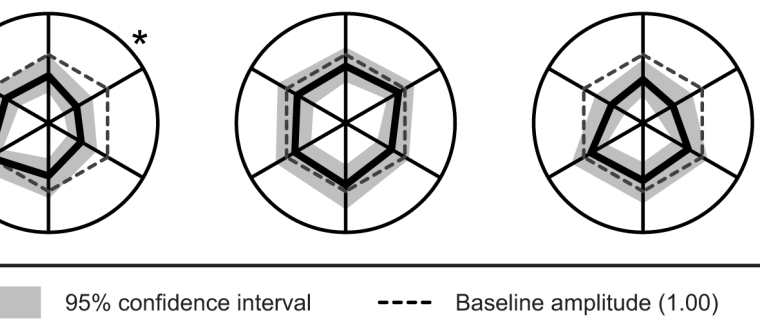

--- Baseline amplitude (1.00)

B
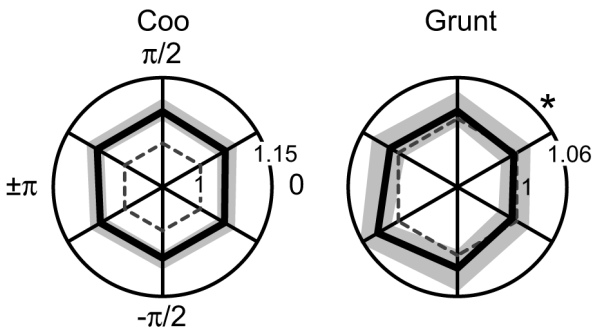

Scream

C

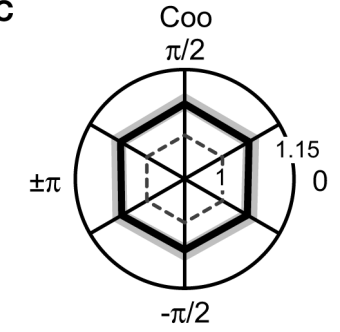

Grunt

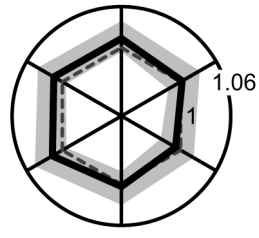

Scream
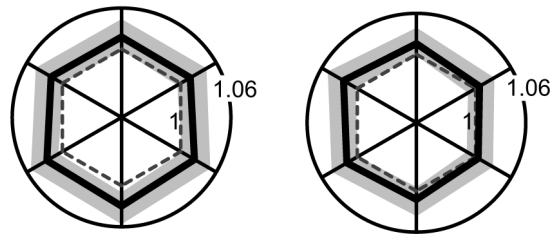

FIGURE 8 |The cross-frequency coupling between the theta phase and gamma-band induced-LFP attenuated with repeated presentations of a vocalization. The data in (A) show the effect that repeated presentations of a vocalization had on the amplitude of the gamma band for each theta phase. The data in each row show the amplitude as a function of vocalization, while the data in the columns illustrate the phase distribution as a function of stimulus presentation. The data in (B) show the relationship between gamma-band amplitude and alpha phase during the first presentation of each vocalization. The data in (C) show the relationship between gamma-band amplitude and beta phase during the first presentation of each vocalization. For all the plots in all three panels, the solid black line shows the mean gamma-amplitude distribution; the gray area surrounding the black line indicates the $95 \%$ confidence interval of this mean distribution; and the dotted line illustrates normalized amplitude is equal to 1 . Asterisks indicate when the gamma-band amplitude is reliably different as a function of the theta phase at $p<0.05$ 
auditory-object processing (Rauschecker and Scott, 2009; Romanski and Averbeck, 2009; Recanzone and Cohen, 2010). Because vocalizations transmit different types of social information such as the vocalizer's emotion, age, and identity (Hauser, 1998; Ghazanfar et al., 2007; Miller and Cohen, 2010), these neural circuits may reflect connectivity with limbic and paralimbic regions of the brain (Gil-da-Costa et al., 2004) as well as other association areas involved in adaptive behavior (Johnson et al., 1996; Pesaran et al., 2008).

\section{CROSS-FREQUENCY COUPLING BETWEEN THETA BAND AND GAMMA BAND}

We found that during the first presentation of a vocalization, the peak of the gamma band of the total-LFP coincided with specific phases of the theta oscillation (Figure 6A). Additionally, these specific theta phases were correlated with the largest gamma-band peak amplitudes, whereas other theta phases corresponded with smaller gamma-band peak amplitudes (Figure 6B). However, with subsequent stimulus presentations, this cross-frequency coupling attenuated. These findings suggest that the cross-frequency coupling between the gamma and theta bands may play a role to differentiate between repeated and novel stimuli.

The high degree of phase-locking of the theta band (see Figures 3D and 5) may help align the gamma band, thereby generating a greater peak gamma-band response. In other words, since the phase-locking of the theta band is highest during the first-stimulus presentation, it is reasonable to speculate that the gamma-band peaks are aligned at specific theta-band phases. Indeed, other studies have also demonstrated that phase-locking in one frequency band can enhance the activity in another frequency band: for example, the entrainment (phase-locking) of delta-band activity by rhythmic stimulus presentations increases the gamma-band response (Lakatos et al., 2005, 2008).

Importantly, this relationship does not simply reflect the simultaneous occurrence of stimulus-evoked gamma- and theta-band phase-locked activity because the gamma-theta coupling was also seen in the induced LFP, which reflects non-phase-locking activity (Figure 8A). Furthermore, since the induced LFP is hypothesized to be related to "high-level cognitive processing" (Tallon-Baudry and Bertrand, 1999), the cross-frequency coupling of induced activity may be related to the processing of the content (e.g., emo-

\section{REFERENCES}

Alain, C., Woods, D. L., and Knight, R. T. (1998). A distributed cortical network for auditory sensory memory in humans. Brain Res. 23, 23-37.

Alho, K., Woods, D. L., Algazi, A., Knight, R. T., and Naatanen, R. (1994). Lesions of frontal cortex diminish the auditory mismatch negativity. Electroencephalogr. Clin. Neurophysiol. 91, 353-362.

Arnal, L. H., Wyart, V., and Giraud, A. L. (2011). Transitions in neural oscillations reflect prediction errors generated in audiovisual speech. Nat. Neurosci. 14, 797-801.
Axmacher, N., Henseler, M. M., Jensen, O., Weinreich, I., Elger, C. E., and Fell, J. (2010). Cross-frequency coupling supports multi-item working memory in the human hippocampus. Proc. Natl. Acad. Sci. U.S.A. 107, 3228-3233.

Baldeweg, T. (2006). Repetition effects to sounds: evidence for predictive coding in the auditory system. Trends Cogn. Sci. (Regul. Ed.) 10, 93-94.

Bokil, H., Purpura, K., Schoffelen, J. M., Thomson, D., and Mitra, P. (2007). Comparing spectra and coherences for groups of unequal size. J. Neurosci. Methods 159, 337-345.

tion, meaning) of the vocalizations (Cheney and Seyfarth, 1990). A second, non-exclusive possibility is that the cross-frequency coupling reflects the salience of the novel stimulus and the monkeys' shift in their attention to this salient stimulus (Gottlieb et al., 1998; Kusunoki et al., 2000) might induce the strong crossfrequency coupling (Gregoriou et al., 2009). This coupling would then attenuate as the salience of the stimulus attenuates due to the repeated stimuli presentation. Thus, our findings may relate to both stimulus salience as well as the detection of the novel stimulus. However, future work is needed to directly correlate cross-frequency coupling in the vPFC with different attributes and demands of a behavioral task (Tort et al., 2008; Siegel et al., 2009).

Finally, similar to previous studies (Lakatos et al., 2005, 2008; Axmacher et al., 2010), we found gamma-theta crossfrequency coupling. However, we could not identify consistent cross-frequency coupling between other frequency bands (Figures 6C,D and 8B,C). It should be noted, however, that crossfrequency coupling may involve the coordinated activity of several bands and not simply two bands as we found in this study. For example, Osipova et al. (2008) demonstrated that gamma-band power covaried with the alpha-band phase but not with the thetaband phase. In addition, Voytek et al. (2010) demonstrated that gamma-band activity was coupled with the theta-band phase in the frontal and temporal cortices during an auditory task. In contrast, the same study also found that gamma-band activity was coupled with the alpha-band phase in the occipital cortex during a visual task. Thus, cross-frequency coupling may be a means by which information can be coded and coordinated within and across different brain regions, and may differ across tasks, stimuli, and brain regions (Bragin et al., 1995; Buzsaki et al., 2003; Lakatos et al., 2005; Tort et al., 2008, 2009; Siegel et al., 2009; Canolty and Knight, 2010; Moldakarimov et al., 2010a,b).

\section{ACKNOWLEDGMENTS}

We would like to thank Asif Ghazanfar for providing stimuli and helpful comments on previous versions of the manuscript, Chandramouli Chandrasekaran for help with the analyses and Jung Hoon Lee, Maria Geffen, and Heather Hersh for helpful comments on the preparation of this manuscript. Yale E. Cohen was supported by grants from the NIDCD-NIH.

Bragin, A., Jando, G., Nadasdy, Z., Hetke, J., Wise, K., and Buzsaki, G. (1995). Gamma (40-100 Hz) oscillation in the hippocampus of the behaving rat. J. Neurosci. 15, 47-60.

Buzsaki, G. (2006). Rhythms of the Brain. New York, NY: Oxford University Press.

Buzsaki, G., Buhl, D. L., Harris, K. D., Csicsvari, J., Czeh, B., and Morozov, A. (2003). Hippocampal network patterns of activity in the mouse. Neuroscience 116, 201-211.

Canolty, R. T., and Knight, R. T. (2010). The functional role of crossfrequency coupling. Trends Cogn. Sci. (Regul. Ed.) 14, 506-515.
Chandrasekaran, C., and Ghazanfar, A. A. (2009). Different neural frequency bands integrate faces and voices differently in the superior temporal sulcus. J. Neurophysiol. 101, 773-788.

Cheney, D. L., and Seyfarth, R. M. (1990). How Monkeys See the World. Chicago, IL: University of Chicago Press.

Cohen, Y. E., Russ, B. E., Davis, S. J., Baker, A. E., Ackelson, A. L., and Nitecki, R. (2009). A functional role for the ventrolateral prefrontal cortex in non-spatial auditory cognition. Proc. Natl. Acad. Sci. U.S.A. 106, 20045-20050. 
Cohen, Y. E., Russ, B. E., Gifford, G. W. III, Kiringoda, R., and MacLean, K. A. (2004). Selectivity for the spatial and nonspatial attributes of auditory stimuli in the ventrolateral prefrontal cortex. J. Neurosci. 24, 11307-11316.

Cohen, Y. E., Theunissen, F., Russ, B. E., and Gill, P. (2007). Acoustic features of rhesus vocalizations and their representation in the ventrolateral prefrontal cortex. J. Neurophysiol. 97, 1470-1484.

Daffner, K. R., Mesulam, M. M., Scinto, L. F., Acar, D., Calvo, V., Faust, R., Chabrerie, A., Kennedy, B. P., and Holcomb, P. J. (2000a). The central role of the prefrontal cortex in directing attention to novel events. Brain 123, 927-939.

Daffner, K. R., Mesulam, M. M., Holcomb, P. J., Calvo, V., Acar, D., Chabrerie, A., Kikinis, R., Jolesz, F. A., Rentz, D. M., and Scinto, L. F. (2000b). Disruption of attention to novel events after frontal lobe injury in humans. J. Neurol. Neurosurg. Psychiatr. 68, 18-24.

Daffner, K. R., Mesulam, M. M., Scinto, L. F., Cohen, L. G., Kennedy, B. P., West, W. C., and Holcomb, P. J. (1998). Regulation of attention to novel stimuli by frontal lobes: an event-related potential study. $\mathrm{Neu}$ roreport 30, 787-791.

Desimone, R. (1996). Neural mechanisms for visual memory and their role in attention. Proc. Natl. Acad. Sci. U.S.A. 93, 13494-13499.

Doeller, C. F., Opitz, B., Mecklinger, A., Krick, C., Reith, W., and Schroger, E. (2003). Prefrontal cortex involvement in preattentive auditory deviance detection: neuroimaging and electrophysiological evidence. Neuroimage 20, 1270-1282.

Fuentemilla, L., Marco-Pallares, J., and Grau, C. (2006). Modulation of spectral power and of phase resetting of EEG contributes differentially to the generation of auditory event-related potentials. Neuroimage 30, 909-916.

Garrido, M. I., Kilner, J. M., Stephan, K. E., and Friston, K. J. (2009). The mismatch negativity: a review of underlying mechanisms. Clin. Neurophysiol. 120, 453-463.

Ghazanfar, A. A., Chandrasekaran, C., and Logothetis, N. K. (2008). Interactions between the superior temporal sulcus and auditory cortex mediate dynamic face/voice integration in rhesus monkeys. J. Neurosci. 28, 4457-4469.

Ghazanfar, A. A., Maier, J. X., Hoffman, K. L., and Logothetis, N. K. (2005). Multisensory integration of dynamic faces and voices in rhesus monkey auditory cortex. J. Neurosci. 25, 5004-5012.

Ghazanfar, A. A., Turesson, H. K., Maier, J. X., van Dinther, R., Patterson, R. D., and Logothetis, N. K. (2007). Vocal-tract resonances as indexical cues in rhesus monkeys. Curr. Biol. 17, 425-430.

Gil-da-Costa, R., Braun, A., Lopes, M., Hauser, M. D., Carson, R. E., Herscovitch, P., and Martin, A. (2004). Toward an evolutionary perspective on conceptual representation: species-specific calls activate visual and affective processing systems in the macaque. Proc. Natl. Acad. Sci. U.S.A. 101, 17516-17521.

Gottlieb, J. P., Kusunoki, M., and Goldberg, M. E. (1998). The representation of visual salience in monkey parietal cortex. Nature 391 , 481-484.

Gouzoules, H., and Gouzoules, S. (2000). Agonistic screams differ among four species of macaques: the significance of motivationstructural rules. Anim. Behav. 59, 501-512.

Gouzoules, H., Gouzoules, S., and Tomaszycki, M. (1998). Agonistic screams and the classification of dominance relationships: are monkeys fuzzy logicians? Anim. Behav. 55, 51-60.

Gouzoules, S., Gouzoules, H., and Marler, P. (1984). Rhesus monkey (Macaca mulatta) screams: representational signalling in the recruitment of agonistic aid. Anim. Behav. 32, 182-193.

Gregoriou, G. G., Gotts, S. J., Zhou, H., and Desimone, R. (2009). Longrange neural coupling through synchronization with attention. Prog. Brain Res. 176, 35-45.

Grill-Spector, K., Henson, R., and Martin, A. (2006). Repetition and the brain: neural models of stimulusspecific effects. Trends Cogn. Sci. (Regul. Ed.) 10, 14-23.

Haenschel, C., Vernon, D. J., Dwivedi, P., Gruzelier, J. H., and Baldeweg, T. (2005). Event-related brain potential correlates of human auditory sensory memory-trace formation. $J$. Neurosci. 25, 10494-10501.

Hauser, M. D. (1998). Functional referents and acoustic similarity: field playback experiments with rhesus monkeys. Anim. Behav. 55, 1647-1658.

Hauser, M. D., and Marler, P. (1993). Food-associated calls in rhesus macaques (Macaca mulatta) II. Costs and benefits of call production and suppression. Behav. Ecol. 4, 206-212.

Hermann, C. S., and Knight, R. T. (2001). Mechanisms of human attention: event-related potentials and oscillations. Neurosci. Behav. Physiol. 25, 465-476.

Jansen, B. H., Agarwal, G., Hegde, A., and Boutros, N. N. (2003). Phase synchronization of the ongoing EEG and auditory EP generation. Clin. Neurophysiol. 114, 79-85.

Johnson, P. B., Ferraina, S., Bianchi, L., and Caminiti, R. (1996). Cortical networks for visual reaching: physiological and anatomical organization of frontal and parietal lobe arm regions. Cereb. Cortex 6, 102-119.

Kayser, C., Petkov, C. I., and Logothetis, N. K. (2008). Visual modulation of neurons in auditory cortex. Cereb. Cortex 18, 1560-1574.

Knight, R. T. (1984). Decreased response to novel stimuli after prefrontal lesions in man. Electroencephalogr. Clin. Neurophysiol. 59, 9-20.

Kusunoki, M., Gottlieb, J., and Goldberg, M. E. (2000). The lateral intraparietal area as a salience map: the representation of abrupt onset, stimulus motion, and task relevance. Vision Res. 40, 1459-1468.

Lakatos, P., Chen, C. M., O'Connell, M. N., Mills, A., and Schroeder, C. E. (2007). Neuronal oscillations and multisensory interaction in primary auditory cortex. Neuron 53 279-292.

Lakatos, P., Karmos, G., Mehta, A D., Ulbert, I., and Schroeder, C. E. (2008). Entrainment of neuronal oscillations as a mechanism of attentional selection. Science 320 , 110-113.

Lakatos, P., O'Connell, M. N., Barczak, A., Mills, A., Javitt, D. C., and Schroeder, C. E. (2009). The leading sense: supramodal control of neurophysiological context by attention. Neuron 64, 419-430.

Lakatos, P., Shah, A. S., Knuth, K. H., Ulbert, I., Karmos, G., and Schroeder, C. E. (2005). An oscillatory hierarchy controlling neuronal excitability and stimulus processing in the auditory cortex. J. Neurophysiol. 94, 1904-1911.

Lee, H., Simpson, G. V., Logothetis, N. K., and Rainer, G. (2005). Phase locking of single neuron activity to theta oscillations during working memory in monkey extrastriate visual cortex. Neuron 45, 147-156.

Lewkowicz, D. J., and Ghazanfar, A. A. (2006). The decline of cross-species intersensory perception in human infants. Proc. Natl. Acad. Sci. U.S.A. 103, 6771-6774

Makeig, S., Debener, S., Onton, J., and Delorme, A. (2004). Mining eventrelated brain dynamics. Trends Cogn. Sci. (Regul. Ed.) 8, 204-210.

Mallat, S. (1999). A Wavelet Tour of Signal Processing. San Diego, CA: Academic Press.
Marler, P., Evans, C. S., and Hauser, M. D. (1992). "Animal signals? Reference, motivation, or both?" in Nonverbal Vocal Communication: Comparative and Developmental Approaches, eds by H. Papouce, $\mathrm{U}$. J. Jürgens, and M. Papoucek (Cambridge: Cambridge University Press), 66-86.

Miller, C. T., and Cohen, Y. E. (2010). "Vocalization processing," in Primate Neuroethology, eds A. Ghazanfar and M. L. Platt (Oxford: Oxford University Press), 237-255.

Miller, E. K., and Cohen, J. D. (2001). An integrative theory of prefrontal cortex function. Annu. Rev. Neurosci. 24, 167-202.

Miller, E. K., Erickson, C. A., and Desimone, R. (1996). Neural mechanisms of visual working memory in prefrontal cortex of the macaque. J. Neurosci. 16, 5154-5167.

Mitra, P. P., and Pesaran, B. (1999). Analysis of dynamic brain imaging data. Biophys. J. 76, 691-708.

Moldakarimov, S., Bazhenov, M., and Sejnowski, T. J. (2010a). Perceptual priming leads to reduction of gamma frequency oscillations. Proc. Natl. Acad. Sci. U.S.A. 107, 5640-5645.

Moldakarimov, S., Bazhenov, M., and Sejnowski, T. J. (2010b). Representation sharpening can explain perceptual priming. Neural Comput. 22, 1312-1332.

Näätänen, R. (1992). Attention and Brain Function. Hillsdale, NJ: Erlbaum.

Osipova, D., Hermes, D., and Jensen, O. (2008). Gamma power is phase locked to posterior alpha activity. PLoS ONE 3, e3990. doi: 10.1371/journal.pone.0003990

Pesaran, B., Nelson, M. J., and Andersen, R. A. (2008). Free choice activates a decision circuit between frontal and parietal cortex. Nature 453, 406-409.

Ranganath, C., and Rainer, G. (2003). Neural mechanisms for detecting and remembering novel events. Nat. Rev. Neurosci. 3, 193-202.

Rauschecker, J. P., and Scott, S. K. (2009). Maps and streams in the auditory cortex: nonhuman primates illuminate human speech processing. Nat. Neurosci. 12, 718-724.

Recanzone, G. H., and Cohen, Y. E. (2010). Serial and parallel processing in the primate auditory cortex revisited. Behav. Brain Res. 5, 1-6.

Rizzuto, D. S., Madsen, J. R., Bromfield, E. B., Schulze-Bonhage, A., and Kahana, M. J. (2006). Human neocortical oscillations exhibit theta phase differences between encoding and retrieval. Neuroimage 31, 1352-1358. 
Rizzuto, D. S., Madsen, J. R., Bromfield, E. B., Schulze-Bonhage, A., Seelig, D., Aschenbrenner-Scheibe, R., and Kahana, M. J. (2003). Reset of human neocortical oscillations during a working memory task. Proc. Natl. Acad. Sci. U.S.A. 100, 7931-7936.

Romanski, L. M., and Averbeck, B. B. (2009). The primate cortical auditory system and neural representation of conspecific vocalizations. Annu. Rev. Neurosci. 32, 315-346.

Romanski, L. M., and Goldman-Rakic, P. S. (2002). An auditory domain in primate prefrontal cortex. Nat. Neurosci. 5, 15-16.

Rosburg, T. (2004). Effects of tone repetition on auditory evoked neuromagnetic fields. Clin. Neurophysiol. 115, 898-905.

Russ, B. E., Orr, L. E., and Cohen, Y. E. (2008). Prefrontal neurons predict choices during an auditory same-different task. Curr. Biol. 18, 1483-1488.

Samar, V. J. (1999). Wavelet analysis of neuroelectric waveforms. Brain Lang. 66, 1-6.
Siegel, M., Warden, M. R., and Miller, E. K. (2009). Phase-dependent neuronal coding of objects in short-term memory. Proc. Natl. Acad. Sci. U.S.A. 15, 21341-21346.

Sinkkonen, J., Tiitinen, H., and Naatanen, R. (1995). Gabor filters: an informative way for analysing eventrelated brain activity. J. Neurosci. Methods 56, 99-104.

Tallon-Baudry, C., and Bertrand, O. (1999). Oscillatory gamma activity in humans and its role in object representation. Trends Cogn. Sci. (Regul. Ed.) 3, 151-162.

Tallon-Baudry, C., Bertrand, O. Delpuech, C., and Pernier, J. (1996). Stimulus specificity of phase-locked and non-phase-locked $40 \mathrm{~Hz}$ visual responses in human. J. Neurosci. 16 4240-4249.

Tort, A. B., Komorowski, R., Eichenbaum, H., and Kopell, N. J. (2010). Measuring phase-amplitude coupling between neuronal oscillations of different frequencies. J. Neurophysiol. 104, 1195-1210.

Tort, A. B., Komorowski, R. W., Manns, J. R., Kopell, N. J., and
Eichenbaum, H. (2009). Thetagamma coupling increases during the learning of item-context associations. Proc. Natl. Acad. Sci. U.S.A. 106, 20942-20947.

Tort, A. B., Kramer, M. A., Thom, C. Gibson, D. J., Kubota, Y., Graybiel, A. M., and Kopell, N. J. (2008). Dynamic cross-frequency couplings of local-field potential oscillations in rat striatum and hippocampus during performance of a T-maze task. Proc. Natl. Acad. Sci. U.S.A. 105, 20517-205122.

Voytek, B., Canolty, R. T., Shestyuk, A., Crone, N. E., Pavizi, J., and Knight, R. T. (2010). Shifts in gamma phase-amplitude coupling frequency from theta to alpha over posterior cortex during visual tasks. Front. Hum. Neurosci. 4:191. doi: 10.3389/fnhum.2010.00191

Zangenehpour, S., Ghazanfar, A. A., Lewkowicz, D. J., and Zatorre R. J. (2009). Heterochrony and cross-species intersensory matching by infant vervet monkeys. PLoS ONE 4, e4302. doi: 10.1371/journal.pone.0004302
Conflict of Interest Statement: The authors declare that the research was conducted in the absence of any commercial or financial relationships that could be construed as a potential conflict of interest.

Received: 11 June 2011; accepted: 18 August 2011; published online: 07 September 2011

Citation: Tsunada J, Baker AE, Christison-Lagay KL, Davis SJ and Cohen YE (2011) Modulation of crossfrequency coupling by novel and repeated stimuli in the primate ventrolateral prefrontal cortex. Front. Psychology 2:217. doi: 10.3389/fpsyg.2011.00217 This article was submitted to Frontiers in Auditory Cognitive Neuroscience, a specialty of Frontiers in Psychology.

Copyright (c) 2011 Tsunada, Baker, Christison-Lagay, Davis and Cohen.

This is an open-access article subject to a non-exclusive license between the authors and Frontiers Media SA, which permits use, distribution and reproduction in other forums, provided the original authors and source are credited and other Frontiers conditions are complied with. 